\title{
ON THE NUMBER OF INVARIANT STRAIGHT LINES FOR POLYNOMIAL DIFFERENTIAL SYSTEMS
}

\author{
Joan C. Artés, Branko Grünbaum and Jaume Llibre
}

If $P$ and $Q$ are two real polynomials in the real variables $x$ and $y$ such that the degree of $P^{2}+Q^{2}$ is $2 n$, then we say that the polynomial differential system $x^{\prime}=P(x, y), y^{\prime}=Q(x, y)$ has degree $n$. Let $\alpha(n)$ be the maximum number of invariant straight lines possible in a polynomial differential systems of degree $n>1$ having finitely many invariant straight lines. In the 1980's the following conjecture circulated among mathematicians working in polynomial differential systems. Conjecture: $\alpha(n)$ is $2 n+1$ if $n$ is even, and $\alpha(n)$ is $2 n+2$ if $n$ is odd. The conjecture was established for $n=2,3,4$. In this paper we prove that, in general, the conjecture is not true for $n>4$. Specifically, we prove that $\alpha(5)=14$. Moreover, we present counterexamples to the conjecture for $n \in\{6,7, \ldots, 20\}$. We also show that $2 n+1 \leq \alpha(n) \leq 3 n-1$ if $n$ is even, and that $2 n+2 \leq \alpha(n) \leq 3 n-1$ if $n$ is odd.

\section{Introduction and statement of the main results.}

Let $P$ and $Q$ be two real polynomials in the real variables $x$ and $y$. We say that the polynomial differential system

$$
x^{\prime}=P(x, y), y^{\prime}=Q(x, y),
$$

has degree $n$ if the degree of the polynomial $P^{2}+Q^{2}$ is $2 n$.

Studies of polynomial differential systems were carried out by Poincaré in $[\mathbf{P 1}],[\mathbf{P} 2]$ and $[\mathbf{P} 3]$. The algebraic feature of polynomial differential systems renders natural certain questions and problems of an algebraic or an algebro-geometric nature, such as to recognize when system (1) has invariant algebraic curves, or is algebraically integrable. See the interesting survey of Schlomiuk $[\mathbf{S c}]$ on these questions. This paper deals with the former aspect.

The straight line $a x+b y+c=0$ is invariant for the flow of system (1), and we call it an invariant straight line of system (1) if $a x^{\prime}+b y^{\prime}=$ $a P(x, y)+b Q(x, y)=(a x+b y+c) R(x, y)$ for some real polynomial $R$.

Suppose that the polynomial differential system (1) of degree $n$ has finitely many invariant straight lines; then we denote by $\alpha(n, P, Q)$ the number 
of invariant straight lines of (1). We define $\alpha(n)$ as the maximum of the $\alpha(n, P, Q)$ when $P$ and $Q$ vary. In the mid-1980's Ye Yanqian told us the following conjecture, circulating among mathematicians working in polynomial differential equations:

\section{Invariant Straight Line Conjecture.}

$$
\alpha(n)= \begin{cases}2 n+1 & \text { for even } n, \\ 2 n+2 & \text { for odd } n .\end{cases}
$$

It is well known that the conjecture is true for $n=2$. Recently Zhang Xikang $[\mathbf{Z h}]$ and J. Sokulski $[\mathbf{S k}]$ proved it for $n$ equal to 3 and 4 . Here we will give shorter proofs of the conjecture for $n=2,3$ (see Corollary 5 ).

The following proposition shows that the bounds in this conjecture could not be decreased; this is formalized in Corollary 2.

Proposition 1. System (1) with

$$
P(x, y)=\prod_{i=1}^{n}(x-i), \quad Q(x, y)=\prod_{i=1}^{n}(y-i),
$$

for $n>1$ has exactly $2 n+1$ (respectively $2 n+2$ ) invariant straight lines if $n$ is even (respectively odd).

Corollary 2. If $n>1$ then

$$
\alpha(n) \geq \begin{cases}2 n+1 & \text { for even } n, \\ 2 n+2 & \text { for odd } n .\end{cases}
$$

The next proposition shows what occurs with the number of invariant straight lines for polynomial differential systems of degree 0 and 1.

Proposition 3. For the polynomial differential system (1) of degree $n$ the following statements hold.

(a) If $n=0$ then (1) has infinitely many invariant straight lines.

(b) If $n=1$ then (1) has either infinitely many invariant straight lines, or at most 2 invariant straight lines.

The next result gives an upper bound of the number of invariant straight lines which a polynomial differential system (1) of degree $n$ having finitely many invariant straight lines can have. This result was also obtained by Zoladek [Zo]. 
Proposition 4. If $n \geq 1$ then $\alpha(n) \leq 3 n-1$.

The next result follows immediately from Corollary 2 and Proposition 4 .

\section{Corollary 5.}

(a) If $n$ is even, then $2 n+1 \leq \alpha(n) \leq 3 n-1$.

(b) If $n$ is odd, then $2 n+2 \leq \alpha(n) \leq 3 n-1$.

(c) The Invariant Straight Line Conjecture is true for $n=2,3$; i.e. $\alpha(2)=$ 5 and $\alpha(3)=8$.

The following result shows some basic results on the invariant straight lines of a polynomial differential system of degree $n$. For the definition of finite and infinite singular points of a polynomial differential system see Section 4 .

Proposition 6. Assume that the polynomial differential system (1) of degree $n$ has finitely many invariant straight lines. Then the following statements hold for system (1).

(a) Either all the points on an invariant straight line are singular or the line contains no more than $n$ singular points.

(b) No more than $n$ invariant straight lines can be parallel.

(c) The set of all invariant straight lines through a single point cannot have more than $n+1$ different slopes.

(d) Either it has infinitely many finite singular points, or it has at most $n^{2}$ finite singular points.

Proposition 6 and the Invariant Straight Line Conjecture induced us to consider the following purely geometric problem.

Given a natural number $n>1$, let $\gamma(n)$ denote the maximum number of straight lines in the real plane satisfying:

(1) Each line in the family has at most $n$ intersection points with the other lines in the family,

(2) no more than $n$ lines in the family can be parallel,

(3) the lines of the family passing through an intersection point cannot have more than $n+1$ different slopes,

(4) the set of all intersection points among lines in the family has cardinality at most $n^{2}$.

Geometric Straight Line Question. Is it true that $\gamma(n)$ is $2 n+1$ (respectively $2 n+2$ ) if $n$ is even (respectively odd)?

Clearly if the Geometric Straight Line Question has a positive answer, then the Invariant Straight Line Conjecture also holds. However, we will 
find examples that answer the Geometric Straight Line Question negatively (see Section 5). These examples will be used to get counterexamples to the Invariant Straight Line Conjecture. More concretely, we have the following two main results.

Theorem 7. The maximum number of invariant straight lines for polynomial differential systems of degree 5 having finitely many invariant straight lines is 14 ; i.e. $\alpha(5)=14$.

Theorem 7 shows that the Invariant Straight Line Conjecture is not true for polynomial differential systems of degree 5. Other counterexamples to the Invariant Straight Line Conjecture are given in the following theorem.

Theorem 8. The following estimates hold.

(a) $15 \leq \alpha(6) \leq 17$.

(b) $18 \leq \alpha(7) \leq 20$.

(c) $19 \leq \alpha(8) \leq 23$.

(d) $24 \leq \alpha(9) \leq 26$.

(e) $25 \leq \alpha(10) \leq 29$.

(f) $30 \leq \alpha(11) \leq 32$.

(g) $31 \leq \alpha(12) \leq 35$.

(h) $32 \leq \alpha(13) \leq 38$.

(i) $33 \leq \alpha(14) \leq 41$.

(j) $36 \leq \alpha(15) \leq 44$.

(k) $37 \leq \alpha(16) \leq 47$.

(l) $38 \leq \alpha(17) \leq 50$.

(m) $39 \leq \alpha(18) \leq 53$.

(n) $42 \leq \alpha(19) \leq 56$.

(o) $43 \leq \alpha(20) \leq 59$.

Remark 9. Darboux in [Da] showed that if a polynomial differential system of degree $n$ has a number of invariant algebraic curves greater than $n(n+1) / 2$, then the system has a first integral. Proposition 4 and the result of Zhang Xikang and Sokulski for $n=4$ show that Darboux's result does not apply for $n \geq 4$ if all the invariant algebraic curves are straight lines (except if the polynomial differential system has infinitely many invariant straight lines).

Remark 10. Suo and Sun in [SS] showed that if a polynomial differential system of degree $n$ has more than $(n-1)(n+2) / 2$ invariant straight lines, 
then the system has no limit cycles. From Proposition 4 it follows that this result does not apply for $n \geq 6$, except if the polynomial differential system has infinitely many invariant straight lines.

The paper is organized as follows. In Section 2 we prove Propositions 1 and 3. Proposition 4 is proved in Section 3. In Section 4 we summarize the Poincaré compactification for a polynomial differential system, and we use it for proving Proposition 6. Section 5 is dedicated to present some simplicial arrangements of lines which provide examples giving a negative answer to the Geometric Straight Line Question. Finally Theorems 7 and 8 are proved in Section 6.

The authors want to express their gratitude to Professor Ye Yanqian for his valuable comments on a preliminary version of this paper.

The first and third authors are partially supported by a DGICYT grant number PB93-0860. The second author was supported in part by NSF Grant DMS-9300657.

\section{Preliminary results.}

The goal of this section is to prove Propositions 1 and 3 .

Proof of Proposition 1. Clearly the $2 n$ straight lines $x=i$ and $y=i$ for $i=1,2, \ldots, n$ are invariant for the flow of the system given in Proposition 1 , and no other horizontal or vertical straight lines are invariant for such a system. Therefore, if the system of Proposition 1 has more invariant straight lines they must have the form $y=a x+b$ with $a \neq 0$, and they must satisfy

$$
\left.\left(y^{\prime}-a x^{\prime}\right)\right|_{y=a x+b} \equiv 0 \text {. }
$$

Therefore

$$
\prod_{i=1}^{n}(a x+b-i)-a \prod_{i=1}^{n}(x-i) \equiv 0
$$

It is easy to see that this identity implies that $a=1$ and $b=0$ if $n$ is even; $a=1$ and $b=0$, or $a=-1$ and $b=n+1$ if $n$ is odd. This completes the proof of Proposition 1.

Proof of Proposition 3. If $n=0$ then statement (a) is immediate.

Suppose $n=1$. If system (1) has no invariant straight lines we are done. If it has at least one invariant straight line, doing a rotation, a translation and a rescaling of the time (if necessary), we can assume that the invariant straight line is $x=0$ and that system (1) can be written in the form

$$
x^{\prime}=x, y^{\prime}=a x+b y+c .
$$


Since the unique invariant straight line of the form $x=$ constant is $x=$ 0 , all the other invariant straight lines (if any exist) must be of the form $y=A x+B$. If $y=A x+B$ is an invariant straight line, then $x(t)=C e^{t}$, $y(t)=A C e^{t}+B$ with $C \neq 0$ must satisfy system (2). Therefore we get

$$
A(1-b)=a \quad \text { and } \quad b B=-c .
$$

If $b=1$ then $a=0$ and system (2) has infinitely many invariant straight lines of the form $y=A x-c$ with $A \in \mathbb{R}$.

If $b=0$ then $c=0$ and system (2) has infinitely many invariant straight lines of the form $y=a x+B$ with $B \in \mathbb{R}$.

Finally, if $b \notin\{0,1\}$ then system (2) has exactly two invariant straight lines, namely

$$
x=0 \quad \text { and } \quad y=\frac{a}{1-b} x-\frac{c}{b} .
$$

\section{An upper bound for $\alpha(n)$.}

In this section we prove Proposition 4.

Proof of Proposition 4. The (signed) curvature of a parametrized planar curve $(x(t), y(t))$ at $t$ is $\left(x^{\prime} y^{\prime \prime}-y^{\prime} x^{\prime \prime}\right)\left(x^{\prime 2}+y^{\prime 2}\right)^{3 / 2}$, where as usual the prime denotes derivative with respect to $t$; for more details see $[\mathbf{C a}]$. If the curvature in all the solutions of system (1) is zero then all solutions of (1) are invariant straight lines. So, since for computing $\alpha(n)$ we must assume that system (1) has finitely many invariant straight lines, the curvature cannot be zero on all the solutions of system (1). Therefore, if $\gamma(t)=(x(t), y(t))$ denotes the solution of (1) through the origin, then doing a translation (if necessary) we can assume that the curvature of $\gamma(t)$ at the origin is different from zero.

First we assume that system (1) has finitely many singular points. Doing a rotation (if necessary), we can assume that all invariant straight lines of system (1) satisfying the hypothesis of Proposition 4 are of the form $y=a x+b$ with $a \neq 0$, and that the system has no singular points on the line $x=0$.

If $y=a x+b$ is an invariant straight line of system (1), we have $Q(x, a x+$ $b)=a P(x, a x+b)$ for all $x \in \mathbb{R}$. In particular, for $x=0$ we get $Q(0, b)=$ $a P(0, b)$. Since system (1) has no singular points on the line $x=0, P(0, b) \neq$ 0 , and consequently

$$
a=\frac{Q(0, b)}{P(0, b)}
$$


Since $y=a x+b$ is an invariant straight line of system (1) its signed curvature is zero, therefore

$$
\begin{gathered}
P(x, a x+b)\left[Q_{x}(x, a x+b) P(x, a x+b)+Q_{y}(x, a x+b) Q(x, a x+b)\right]- \\
Q(x, a x+b)\left[P_{x}(x, a x+b) P(x, a x+b)+P_{y}(x, a x+b) Q(x, a x+b)\right]=0
\end{gathered}
$$

for all $x \in \mathbb{R}$. In particular, for $x=0$ we get

$$
P^{2}(0, b) Q_{x}(0, b)+P(0, b) Q(0, b)\left[Q_{y}(0, b)-P_{x}(0, b)\right]-Q^{2}(0, b) P_{y}(0, b)=0 .
$$

We remark that this expression is not zero for $b=0$, otherwise the curvature of $\gamma(t)$ at the origin will be zero. So, if $y=a x+b$ is an invariant straight line, then $b$ must be a root of a polynomial of degree at most $3 n-1$. Hence, since $a$ is determined from (5) it follows that the number of invariant straight lines of system (1) is at most $3 n-1$.

Now we assume that system (1) has infinitely many singular points. Therefore the polynomials $P$ and $Q$ have a common polynomial factor $R$ of degree $m$ such that the points $(x, y)$ of the plane satisfying $R(x, y)=0$ are singular points, and by Bezout Theorem in the complement of the algebraic curve $R(x, y)=0$ system (1) has at most $(n-m)^{2}$ singular points. Hence the system

$$
x^{\prime}=P(x, y) / R(x, y), y^{\prime}=Q(x, y) / R(x, y),
$$

has finitely many singular points. Repeating the previous arguments we get that this system has at most $3(n-m)-1$ invariant straight lines. So system (1) has at most $3(n-m)-1+m<3 n-1$ invariant straight lines. Consequently, the proposition is proved.

\section{Poincaré compactification and basic results.}

Let $X=(P, Q)$ be the vector field associated to system (1) of degree $n$. The Poincaré compactified vector field $p(X)$ corresponding to $X$ is a vector field induced on the two-dimensional sphere $\mathbb{S}^{2}$ as follows. Let $\mathbb{S}^{2}=\{y=$ $\left.\left(y_{1}, y_{2}, y_{3}\right) \in \mathbb{R}^{3}: y_{1}^{2}+y_{2}^{2}+y_{3}^{2}=1\right\}$ (the Poincaré sphere) and $T_{y^{2}} \mathbb{S}^{2}$ the tangent space to $\mathbb{S}^{2}$ at point $y$. Consider the central projections $f_{+}: T_{(0,0,1)} \mathbb{S}^{2} \rightarrow \mathbb{S}_{+}^{2}=$ $\left\{y \in \mathbb{S}^{2}: y_{3}>0\right\}$ and $f_{-}: T_{(0,0,1)} \mathbb{S}^{2} \rightarrow \mathbb{S}_{-}^{2}=\left\{y \in \mathbb{S}^{2}: y_{3}<0\right\}$. These maps define two copies of $X$, one in the northern hemisphere and the other in the southern hemisphere. Denote by $X^{\prime}$ the vector field defined on $\mathbb{S}^{2}$ except on its equator $\mathbb{S}^{1}=\left\{y \in \mathbb{S}^{2}: y_{3}=0\right\}$ by $D f_{+} \circ X$ and $D f_{-} \circ X$. Clearly $\mathbb{S}^{1}$ is identified to the infinity of $\mathbb{R}^{2}$. In order to extend $X^{\prime}$ to an analytic vector field on $\mathbb{S}^{2}$ (including $\mathbb{S}^{1}$ ) it is necessary that $X$ satisfies suitable hypotheses. Since the degree of $X$ is $n$, the Poincaré compactification $p(X)$ of $X$ is the 
only analytic extension of $y_{3}^{n-1} X^{\prime}$ to $\mathbb{S}^{2}$. For the flow of the compactified vector field $p(X)$, the equator $\mathbb{S}^{1}$ is invariant. On $\mathbb{S}^{2} \backslash \mathbb{S}^{1}$ there are two symmetric copies of $X$, and knowing the behavior of $p(X)$ around $\mathbb{S}^{1}$, we know the behavior of $X$ at infinity. The projection of the closed northern hemisphere of $\mathbb{S}^{2}$ on $y_{3}=0$ under $\left(y_{1}, y_{2}, y_{3}\right) \mapsto\left(y_{1}, y_{2}\right)$ is called the Poincaré disc.

As $\mathbb{S}^{2}$ is a differentiable manifold for computing the expression of $p(X)$, we can consider the following six local charts $U_{i}=\left\{y \in \mathbb{S}^{2}: y_{i}>0\right\}$, and $V_{i}=$ $\left\{y \in \mathbb{S}^{2}: y_{i}<0\right\}$ where $i=1,2,3$, and the diffeomorphisms $F_{i}: U_{i} \rightarrow \mathbb{R}^{2}$ and $G_{i}: V_{i} \rightarrow \mathbb{R}^{2}$ which are the inverses of the central projections from the vertical planes tangents at points $(1,0,0),(-1,0,0),(0,1,0),(0,-1,0),(0,0,1)$, $(0,0,-1)$ respectively. We denote by $z=\left(z_{1}, z_{2}\right)$ the value of $F_{i}(y)$ or $G_{i}(y)$ for any $i=1,2,3$. So $z$ represents different things according to the local chart under consideration. In these coordinates and in the local charts $U_{i}$ and $V_{i}$ for $i=1,2, z_{2}=0$ denotes always the points of $\mathbb{S}^{1}$.

A singular point $q$ of $p(X)$ is called an infinite (respectively finite) singular point of $X$ if it is a singular point of $p(X)$ in $\mathbb{S}^{1}$ (respectively $\mathbb{S}^{2} \backslash \mathbb{S}^{1}$ ). The infinite singular points of $X$ are the points $\left(z_{1}, 0\right)$ satisfying

$$
\begin{array}{ll}
F\left(z_{1}\right)=Q_{n}\left(1, z_{1}\right)-z_{1} P_{n}\left(1, z_{1}\right)=0 & \text { if }\left(z_{1}, 0\right) \in U_{1}, \\
G\left(z_{1}\right)=P_{n}\left(z_{1}, 1\right)-z_{1} Q_{n}\left(z_{1}, 1\right)=0 & \text { if }\left(z_{1}, 0\right) \in U_{2},
\end{array}
$$

where $P_{n}$ and $Q_{n}$ are the homogeneous part of degree $n$ of $P$ and $Q$. For more details on the Poincaré compactification, see, for instance $[\mathbf{G o}]$, $[\mathbf{S o}]$ and $[$ ALGM].

The main goal of this section is to prove Proposition 6. That is, to obtain basic results on the set of invariant straight lines of a polynomial differential system of a given degree.

Proof of Proposition 6. Suppose that system (1) satisfies the hypothesis of Proposition 6.

If system (1) has an invariant straight line, performing (if necessary) a rotation and a translation in the plane $(x, y)$, we can assume that the invariant straight line has the equation $x=0$. The singular points $(0, y)$ of system (1) on this invariant straight line must satisfy $Q(0, y)=0$. So, if $Q(0, y) \equiv 0$ then $x=0$ is formed by singular points, otherwise the polynomial $Q(0, y)$ of degree at most $n$ has at most $n$ different real roots. Therefore, statement (a) is proved.

In order to show statement (b) we assume that system (1) has $k$ invariant parallel straight lines. After a rotation in the plane $(x, y)$ (if necessary), the $k$ parallel straight lines have equation of the form $x=a_{i}$ for $i=1,2, \ldots, k$. Then, since $x-a_{i}=0$ is invariant, it follows that $x-a_{i}$ divides $P(x, y)$. 
Since the degree of $P$ is at most $n$, it follows that $k \leq n$. Hence, statement (b) is proved.

We will prove statement (c) in two steps. First we assume that $F\left(z_{1}\right)$ in (6) is not identically zero. Performing a rotation (if necessary) we can assume that system (1) has no invariant straight lines of the form $x=$ constant. Then, each invariant straight line provides two pairs of diametrically opposite infinite singular points, and all these pairs are contained in the local charts $U_{1}$ and $V_{1}$. Moreover, if $\left(z_{1}, 0\right)$ is the infinite singular point of the local chart $U_{1}$ associated to the invariant straight line $y=a x+b$, then $a=z_{1}$. Since the infinite singular point $\left(z_{1}, 0\right)$ of the local chart $U_{1}$ must satisfy Equation (6), and $F\left(z_{1}\right)$ is a polynomial of degree at most $n+1$, it follows that the set of all invariant straight lines of system (1) cannot have more than $n+1$ different slopes. Therefore, statement (c) follows.

If $F\left(z_{1}\right) \equiv 0$ in (6) then we consider that a point belongs to $m>n+1$ invariant straight lines. We move this point to the origin with a translation and one invariant straight line to $x=0$ with a rotation (if necessary). If we consider now the vector field in the local chart $U_{1}$, we will have a polynomial differential system of degree $n+1$ with $z_{2}=0$ being a common factor. If we remove this factor, the system has degree $n$ at most. The set of straight lines crossing the origin now are $m-1$ parallel lines of the form $z_{1}=$ constant. By statement (b) this set has at most $n$ invariant straight lines and we get a contradiction with the assumption $m>n+1$. This completes the proof of statement (c).

Statement (d) follows immediately from Bezout Theorem.

\section{On the Geometric Straight Line Question.}

By an arrangement of $n$ lines $A(n)$ we mean the cell complex determined in the real projective plane by a finite family of $n$ straight lines which do not have a common point. We call an arrangement of lines $A(n)$ simplicial provided all the faces of $A$ are simplices. For more details see $[\mathbf{G r}],[\mathbf{C F}]$ and $[\mathbf{S p}]$.

In $[\mathbf{G r}]$ Grünbaum presented a catalogue of simplicial arrangements of lines. The catalogue lists all the known isomorphism-types of simplicial arrangements of straight lines in the real projective plane, and provides illustrations of those types which cannot be easily described. From this catalogue we can obtain the following examples that answer the Geometric Straight Line Question in the negative sense. For those n's that we have several examples we only present one with the maximal number of straight lines.

Figure 1 shows the simplicial arrangement $A(15)$ of the following 14 straight 
lines in the affine real plane plus the line of infinity:

$$
\begin{aligned}
& x=i, \\
& x=i(2 k-1), \\
& y=i, \\
& y=i(2 k-1), \\
& y=i x, \\
& y=-k x+i(1-k), \\
& y=-\frac{1}{k} x+i \frac{1-k}{k},
\end{aligned}
$$

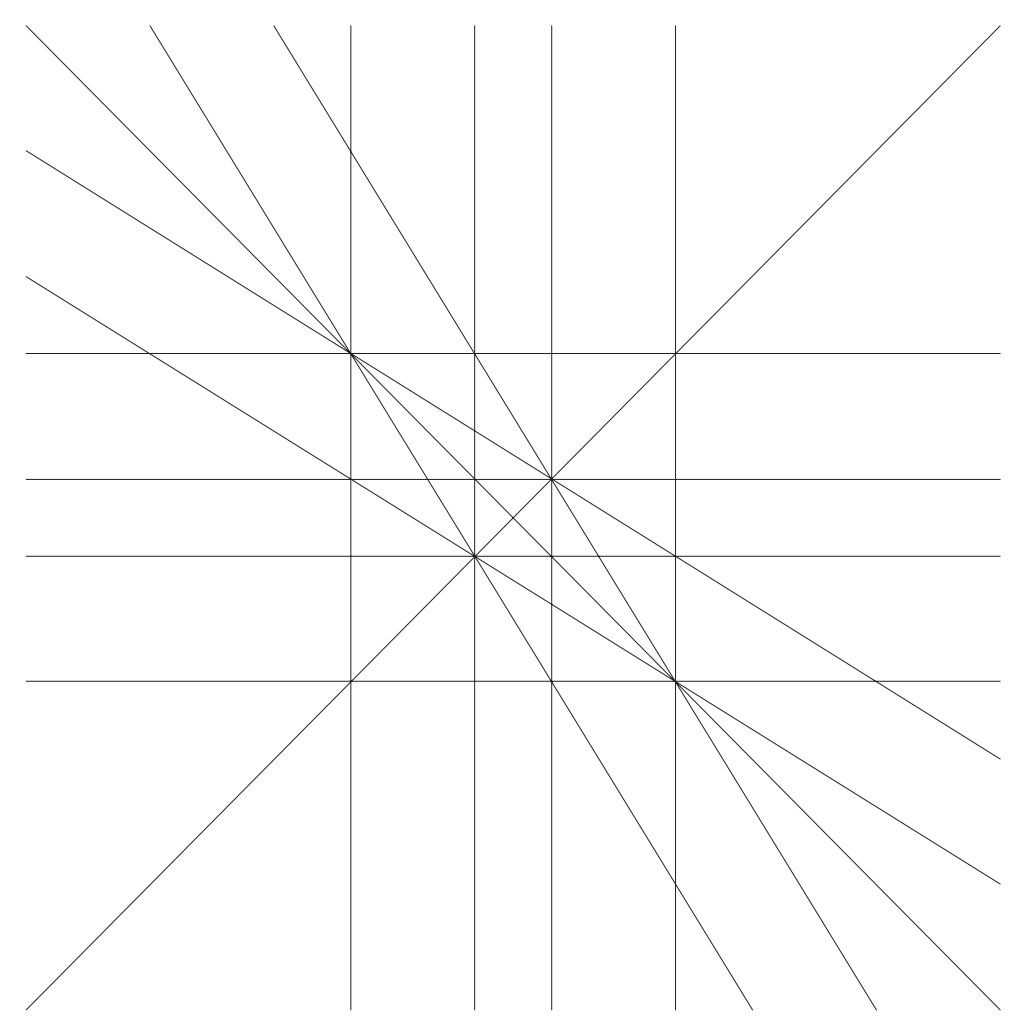

Figure 1. The simplicial arrangement $A(15)$. 
where $i \in\{-1,1\}$ and $k$ denotes the golden section, i.e. $k=(\sqrt{5}-1) / 2$. This arrangement shows that $\gamma(5) \geq 14$, and hence provides a negative answer to the Geometric Straight Line Question for $n=5$. It corresponds to the arrangement $A_{1}^{*}(15)$ in the notation of $[\mathbf{G r}]$.

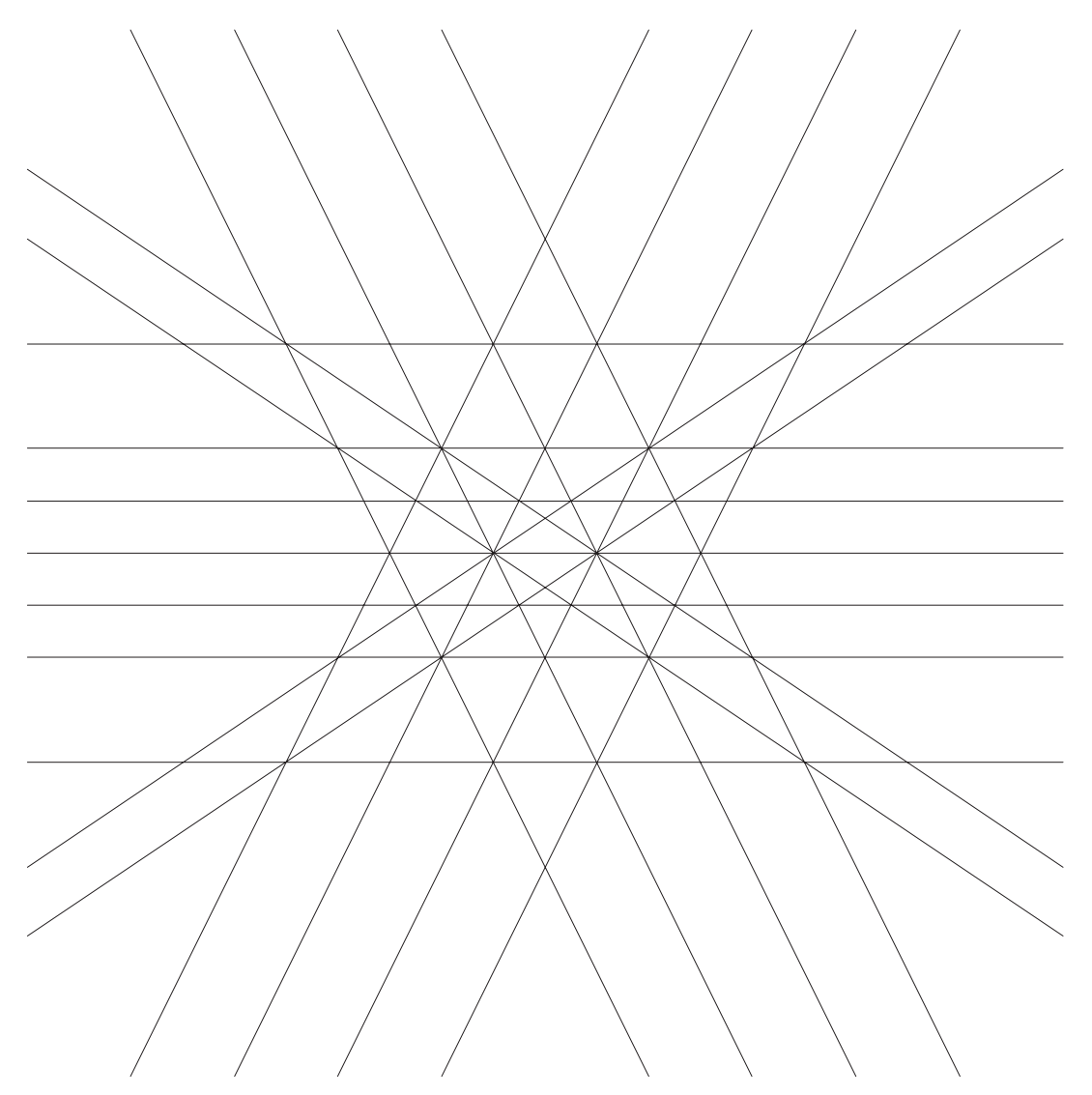

Figure 2. The simplicial arrangement $A(20)$.

Figure 2 shows the simplicial arrangement $A(20)$ of the following 19 straight lines in the affine real plane plus the line of infinity:

$$
\begin{aligned}
& y=j, \\
& y=2 i x+l, \\
& y=\frac{2}{3}(i x+m),
\end{aligned}
$$


where $i, m \in\{-1,1\}, j=-4,-2,-1,0,1,2,4$ and $l=-6,-2,2,6$. This arrangement shows that $\gamma(8) \geq 19$ and provides a negative answer to the Geometric Straight Line Question for $n=8$. It corresponds to the arrangement $A_{5}^{*}(20)$ in the notation of $[\mathbf{G r}]$.

Figure 3 shows the simplicial arrangement $A(25)$ of the following 24 straight lines in the affine real plane plus the line of infinity:

$$
\begin{aligned}
& x=j, \\
& y=j, \\
& y=i(\sqrt{2}+1) x, \\
& y=i(\sqrt{2}-1) x, \\
& y=i x+l,
\end{aligned}
$$

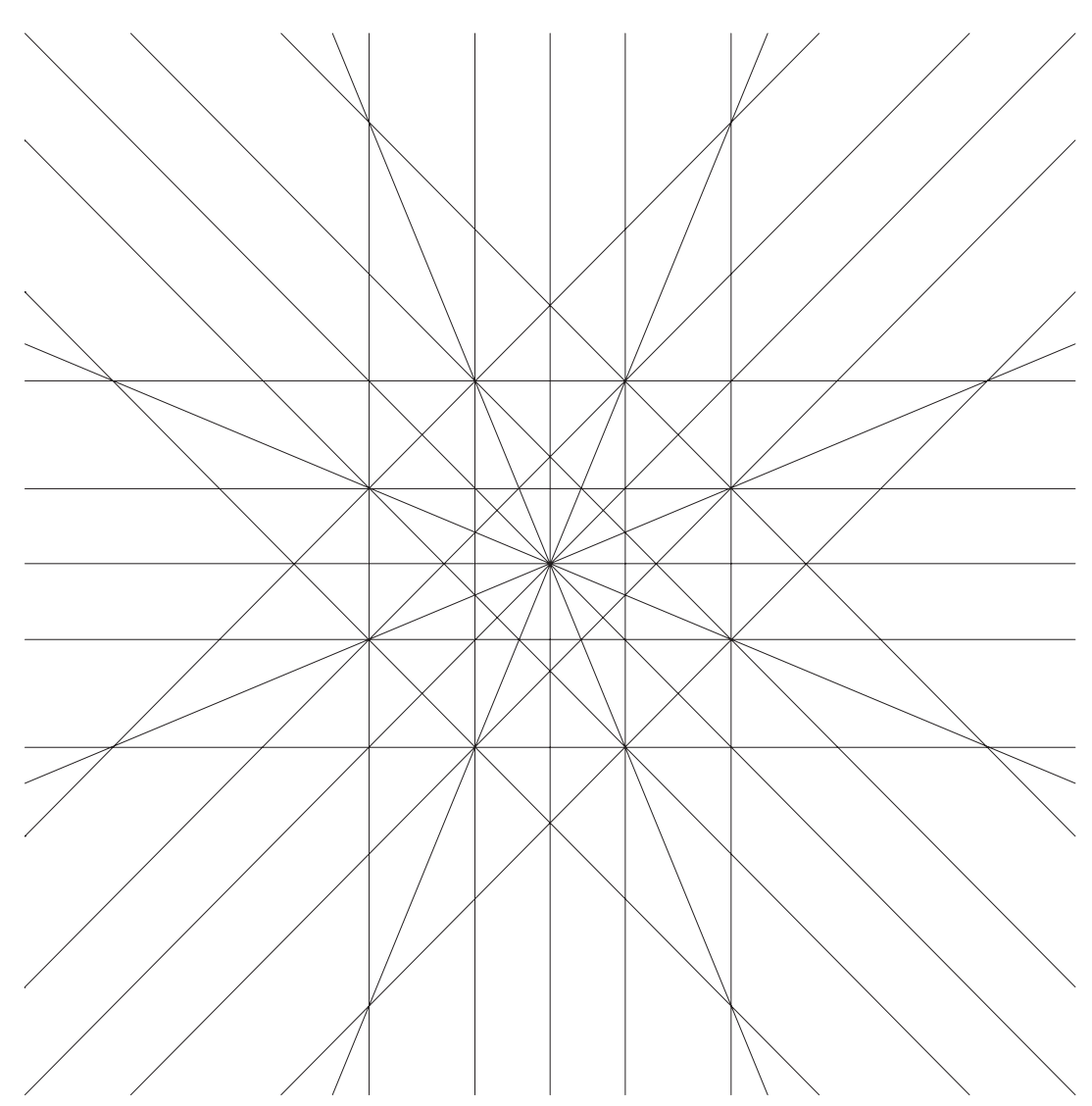

Figure 3. The simplicial arrangement $A(25)$. 
where $j=-(\sqrt{2}+1),-1,0,1, \sqrt{2}+1, i \in\{-1,1\}$ and $l=-(\sqrt{2}+2),-\sqrt{2}, 0$, $\sqrt{2}, \sqrt{2}+2$. This arrangement shows that $\gamma(9) \geq 24$, and provides a negative answer to the Geometric Straight Line Question for $n=9$. It corresponds to the arrangement $A_{5}^{*}(25)$ in the notation of $[\mathbf{G r}]$.

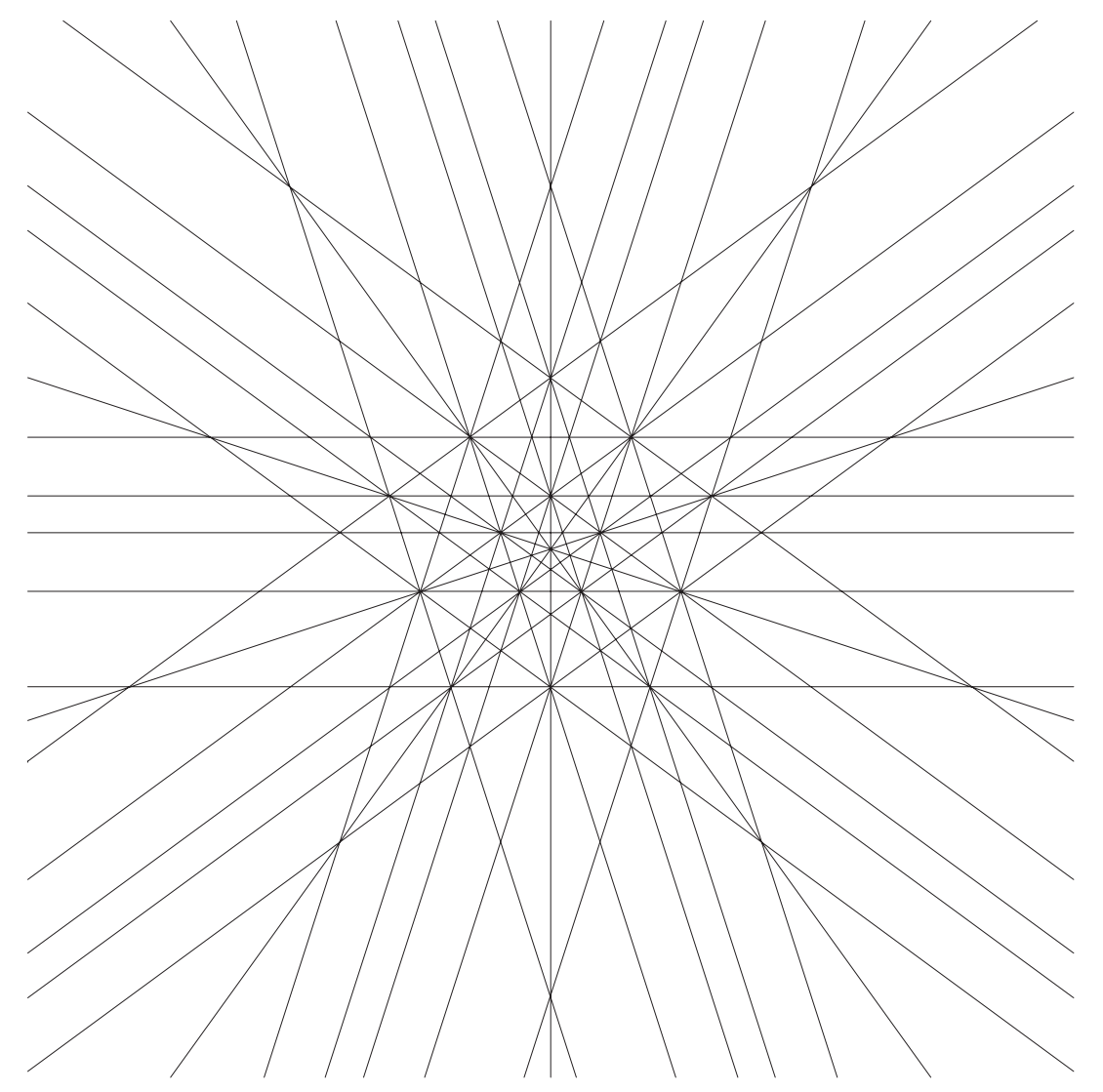

Figure 4. The simplicial arrangement $A(31)$.

Figure 4 shows the simplicial arrangement $A(31)$ with 30 straight lines in the affine real plane plus the line of infinity. The 30 straight lines are obtained by rotating the following 6 straight lines:

$$
\begin{aligned}
& x=0, \\
& y=1,
\end{aligned}
$$




$$
\begin{aligned}
& y=\cos (2 \pi / 5), \\
& y=\cos (4 \pi / 5), \\
& y=\frac{\cos (2 \pi / 5)+1}{2 \cos (2 \pi / 5)}, \\
& y=-\frac{\cos (\pi / 5)}{\cos (2 \pi / 5)},
\end{aligned}
$$

by angles of $2 \pi / 5,4 \pi / 5,6 \pi / 5$ and $8 \pi / 5$. This arrangement shows that $\gamma(11) \geq 30$ and provides a negative answer to the Geometric Straight Line Question for $n=11$. It corresponds to the arrangement $A_{2}^{*}(31)$ of page 96 in the notation of $[\mathbf{G r}]$.

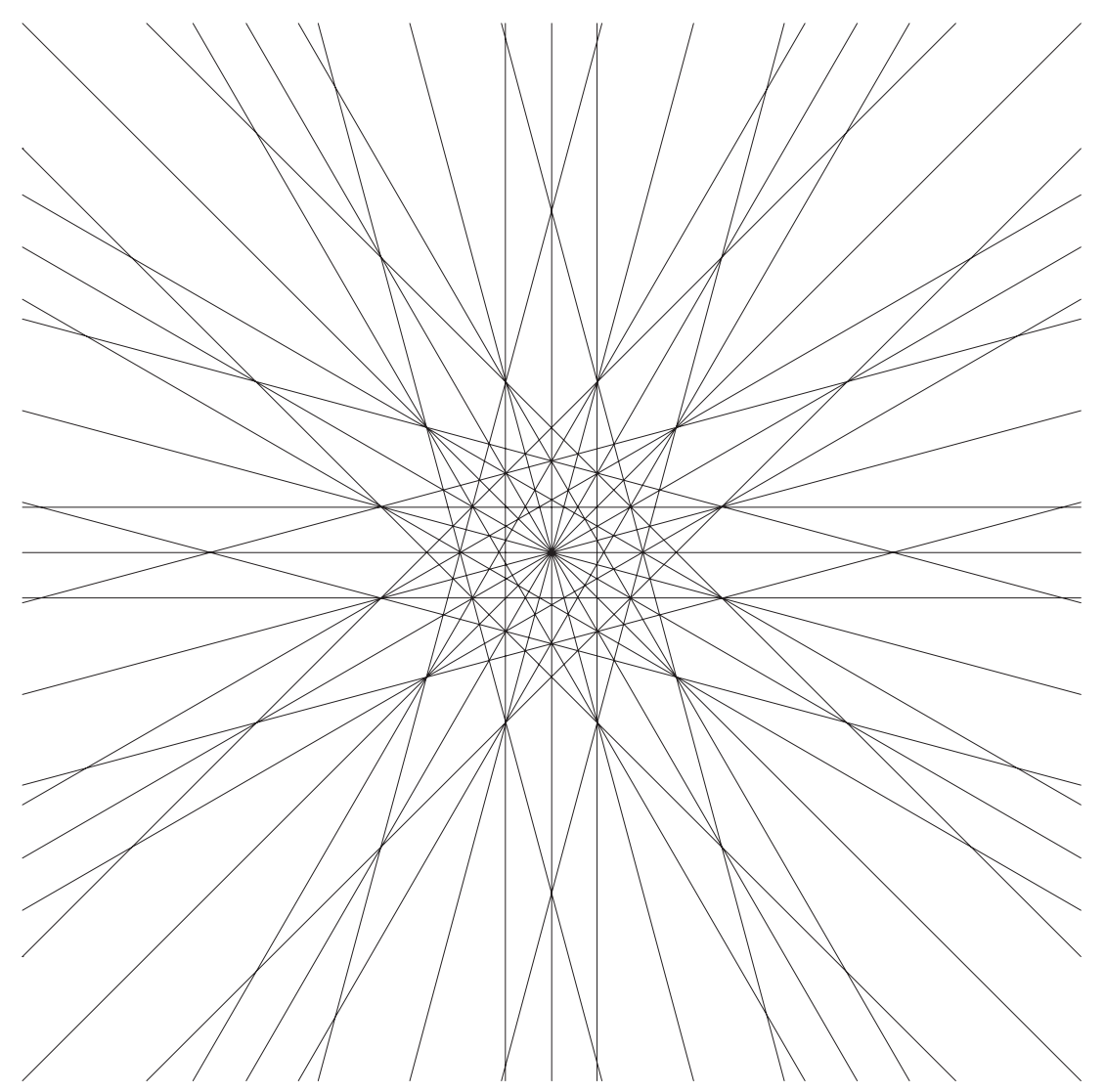

Figure 5. The simplicial arrangement $A(37)$.

Figure 5 shows the simplicial arrangement $A(37)$ with 36 straight lines in the affine real plane plus the line of infinity. The 36 straight lines are 
obtained by rotating the following 12 straight lines:

$$
\begin{aligned}
& x=0, \\
& x=i \sin (\pi / 12), \\
& y=0, \\
& y=i \sin (\pi / 12), \\
& y=i x, \\
& y=i x+j(\cos (\pi / 12)-\sin (\pi / 12)),
\end{aligned}
$$

by angles of $\pi / 6$ and $\pi / 3$. Here $i, j \in\{-1,1\}$. This arrangement shows that $\gamma(15) \geq 36$ and provides a negative answer to the Geometric Straight Line Question for $n=15$. It corresponds to the arrangement $A_{2}^{*}(37)$ in the notation of $[\mathbf{G r}]$.

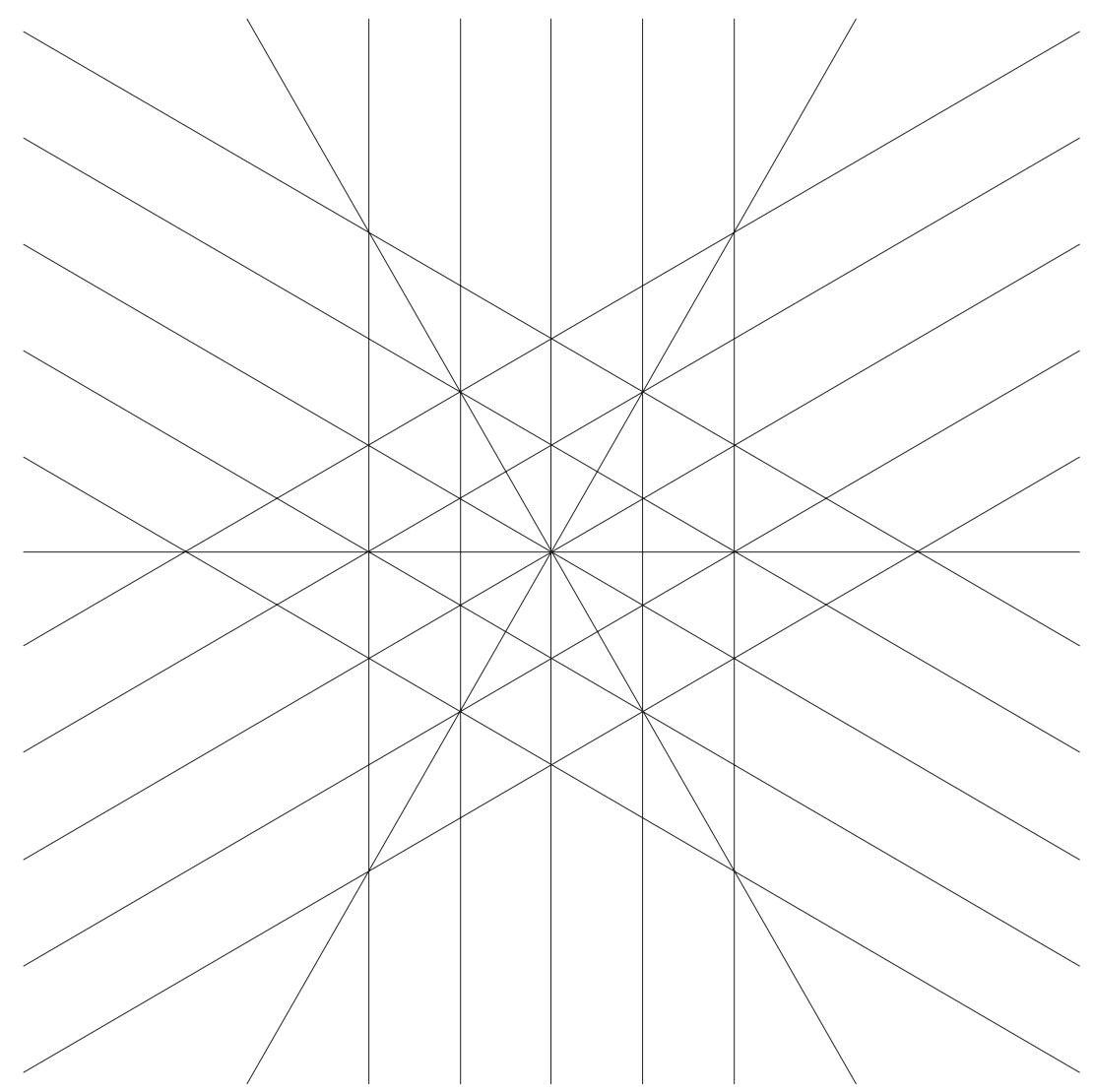

Figure 6. The simplicial arrangement $A(19)$. 
As far as we know only one new simplicial arrangement of lines has been found after the publication of the catalogue of $[\mathbf{G r}]$, which is not relevant here because it does not provide a negative answer to the Geometric Straight Line Question.

In $[\mathbf{G r}]$ three infinite families of simplicial arrangements of lines are presented. But none of these arrangements provides a negative answer to the Geometric Straight Line Question. Here we find a new infinite family of arrangements of lines which leads to a negative answer to the Geometric Straight Line Question for infinitely many n's.

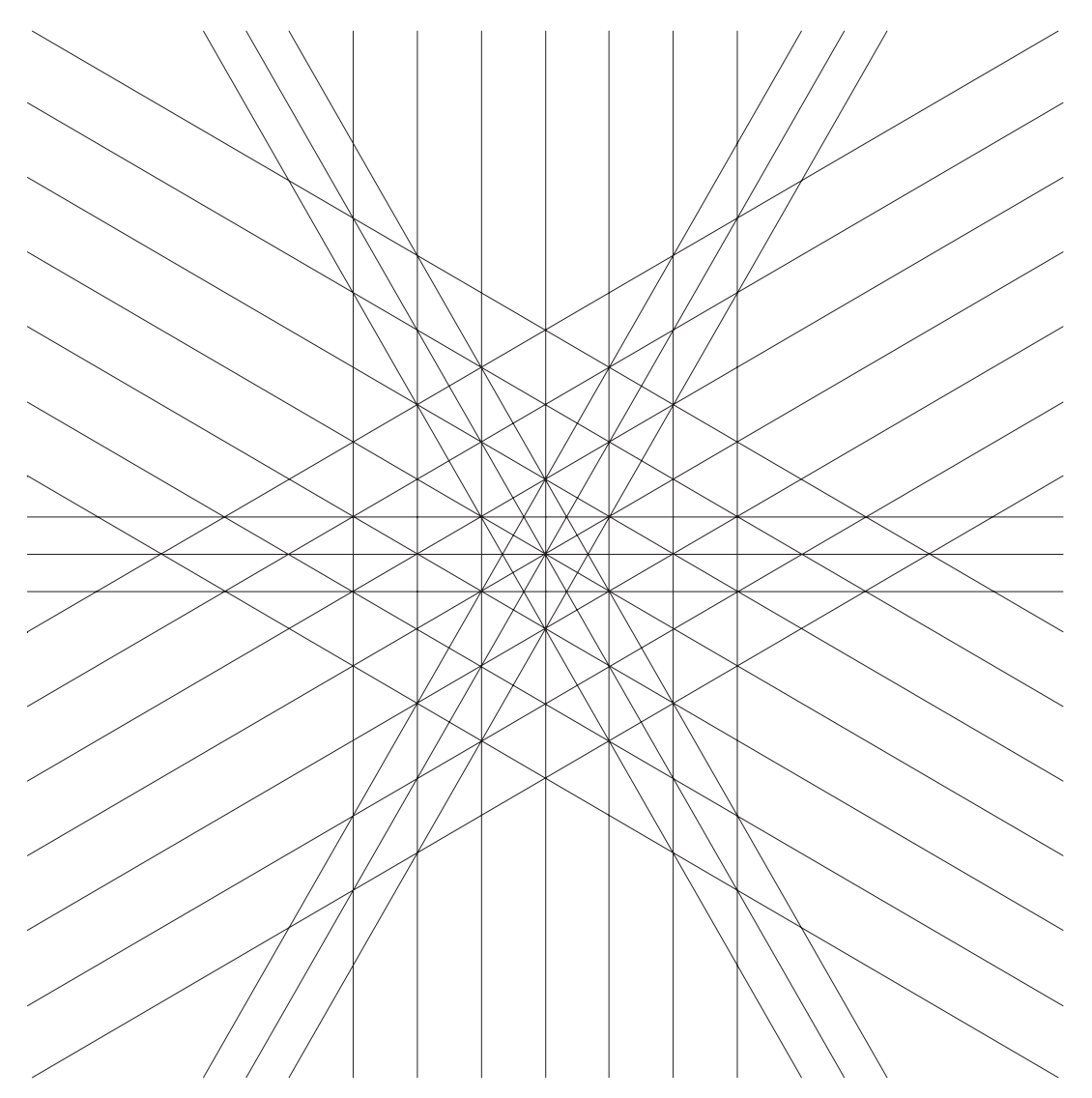

Figure 7. The simplicial arrangement $A^{\prime}(31)$.

The new family $A(2 n+5)$ with $n=6 k+1$ and $k=1,2, \ldots$ is formed by the line at infinity plus the following set of $2 n+4$ straight lines in the affine 
real plane:

$$
\begin{aligned}
& x=\frac{\sqrt{3}}{2} i, \\
& y=\frac{1}{\sqrt{3}} x+i, \\
& y=-\frac{1}{\sqrt{3}} x+i, \\
& y=\frac{j}{2}, \\
& y=\sqrt{3} x+j, \\
& y=-\sqrt{3} x+j,
\end{aligned}
$$

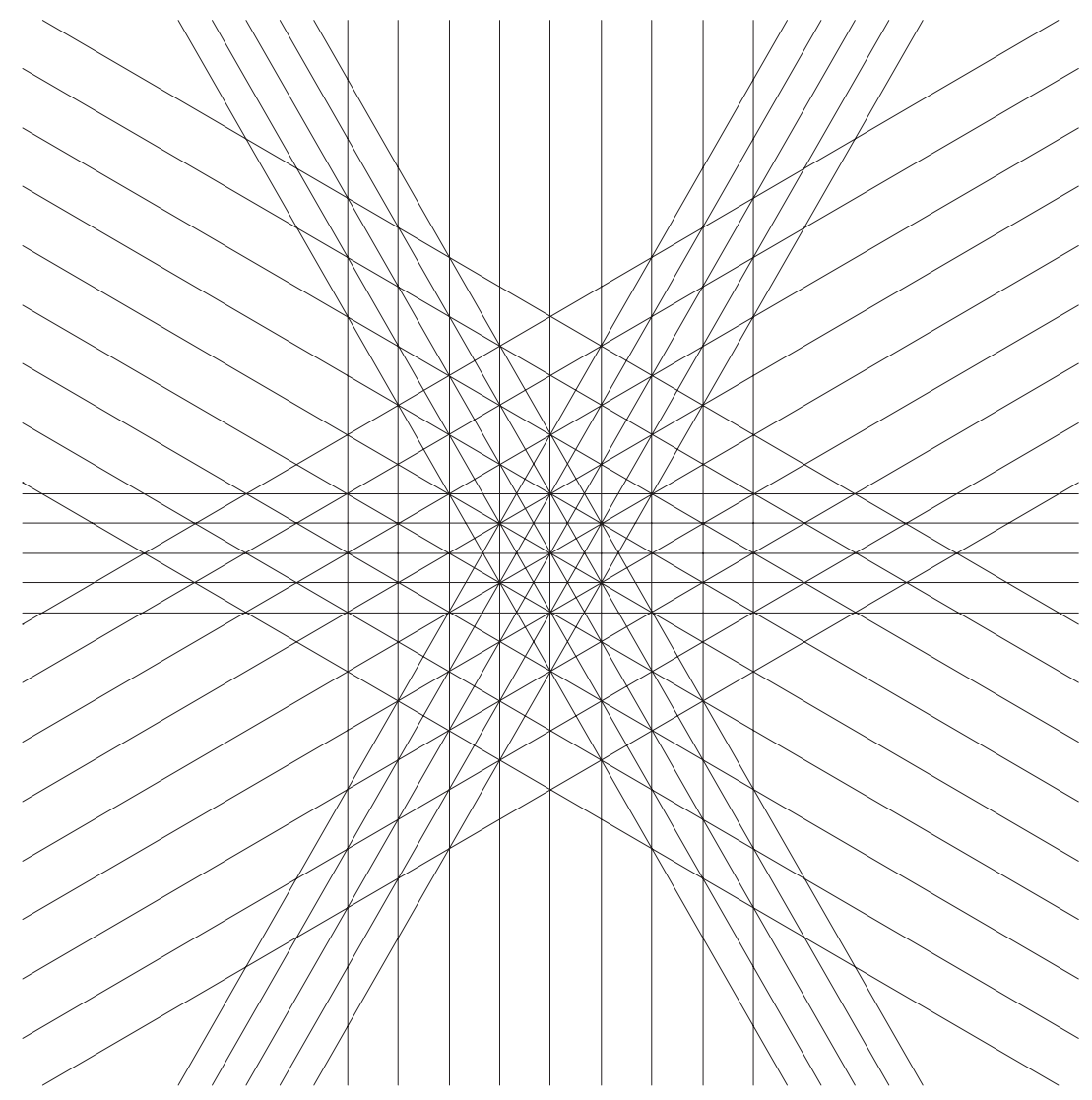

Figure 8. The simplicial arrangement $A(43)$. 
where $i=-(k+1),-k, \ldots,-1,0,1, \ldots, k, k+1$ and $j=-(k-1),-(k-$ 2 ) $, \ldots,-1,0,1, \ldots, k-2, k-1$. We remark that the cases $n=7,13$ (see Figures 6 and 7, respectively) correspond to simplicial arrangements that already appeared in the catalogue of $[\mathbf{G r}]$ with the notation $A_{1}^{*}(19)$ and $A_{2}^{*}(31)$ of page 105 , respectively. The arrangements of lines of this family for $n=19,25,31, \ldots$ are not simplicial, see for instance the arrangement with $n=19$ in Figure 8 . The arrangement $A(2 n+5)$ shows that $\gamma(6 k+1) \geq$ $12 k+7$, and so provides a negative answer to the Geometric Straight Line Question for $n=6 k+1$ and $k=1,2, \ldots$.

\section{Counterexamples to the Invariant Straight Line Conjecture.}

In this section we prove Theorems 7 and 8 . We will need the following auxiliary result.

Lemma 9. If there exists a polynomial differential system (1) of degree $n$ with $k$ invariant straight lines, then there exists a polynomial differential system of degree $n+1$ with $k+1$ invariant straight lines.

Proof. Clearly the polynomial differential system

$$
x^{\prime}=(a x+b y+c) P(x, y), y^{\prime}=(a x+b y+c) Q(x, y),
$$

where $a^{2}+b^{2} \neq 0$ and the straight line $a x+b y+c=0$ is different from the invariant straight lines of system (1), is of degree $n+1$ and has the $k$ invariant straight lines of system (1) plus the invariant straight line $a x+b y+c=0$ formed by singular points.

Now we compute the polynomial differential system of least degree which has as invariant straight lines the straight lines in the affine real plane of the arrangements of Figures 1 to 8 . Thus if we want to find a polynomial differential system which has as invariant straight lines the straight lines of Figure 1, by Proposition 6 the degree of such a system must be at least 5 . Then, in order that such a polynomial differential system has the invariant straight lines $x=i, x=i(2 k-1), y=i$ and $y=i(2 k-1)$ with $i \in\{-1,1\}$ and $k=(\sqrt{5}-1) / 2$, clearly it must be of the form:

$$
\begin{aligned}
& x^{\prime}=\left(x^{2}-1\right)\left(x^{2}-(2 k-1)^{2}\right) P_{1}(x, y), \\
& y^{\prime}=\left(y^{2}-1\right)\left(y^{2}-(2 k-1)^{2}\right) Q_{1}(x, y),
\end{aligned}
$$

where $P_{1}(x, y)$ and $Q_{1}(x, y)$ are arbitrary real polynomials of degree 1 in the variables $x$ and $y$. Now we must determine the 6 coefficients of $P_{1}$ and $Q_{1}$ in 
such a way that the above polynomial differential system has the following 6 invariant straight lines

$$
y=i x, y=-k x+i(1-k) \text { and } y=-\frac{1}{k} x+i \frac{1-k}{k} .
$$

This is equivalent to solve a linear system in the coefficients of the polynomials $P_{1}$ and $Q_{1}$. In order to solve this linear system and the ones for the other cases we have used the program MATHEMATICA. In the easier cases the program has been able to solve the global system. In the more complicated ones we have needed to work interactively with the program and solve the system by a sequence of steps. The linear system has a unique solution depending on one parameter. This parameter can be omitted in the polynomial differential system by a rescaling of the time variable. Hence the polynomial differential system of degree 5 which has as invariant straight lines those of Figure 1 is:

$$
\begin{aligned}
& x^{\prime}=\left(x^{2}-1\right)\left(x^{2}-(\sqrt{5}-2)^{2}\right)(x+\sqrt{5} y), \\
& y^{\prime}=\left(y^{2}-1\right)\left(y^{2}-(\sqrt{5}-2)^{2}\right)(\sqrt{5} x+y) .
\end{aligned}
$$

Proof of Theorem 7. From Proposition 4 and since the above polynomial differential system of degree 5 has 14 invariant straight lines, Theorem 7 follows.

Now we want to find a polynomial differential system of degree 7 which has as invariant straight lines the straight lines of Figure 6. Working as in the case of Figure 1, we find that such a polynomial differential system is:

$$
\begin{aligned}
x^{\prime}= & 2 x\left(x^{2}-3\right)\left(4 x^{2}-3\right)\left(x^{2}+21 y^{2}-12\right), \\
y^{\prime}= & y\left(-216+378 x^{2}+378 y^{2}-315 x^{4}-189 y^{4}\right. \\
& \left.+35 x^{6}+105 x^{4} y^{2}-63 x^{2} y^{4}+27 y^{6}\right) .
\end{aligned}
$$

In order to find a polynomial differential system of degree 8 which has as invariant straight lines the straight lines of Figure 2, working as in the case of Figure 1, we see that such a polynomial differential system is:

$$
\begin{aligned}
x^{\prime}= & 80640-11264 x^{2}-224000 y^{2}-89600 x^{4}+281344 x^{2} y^{2}+101920 y^{4} \\
& +21504 x^{6}-62720 x^{4} y^{2}-15680 x^{2} y^{4}-14000 y^{6} \\
& -1280 x^{8}+5376 x^{6} y^{2}-5600 x^{4} y^{4}+3920 x^{2} y^{6}+315 y^{8} \\
y^{\prime}= & 4096 x y\left(y^{2}-1\right)\left(y^{2}-4\right)\left(y^{2}-16\right) .
\end{aligned}
$$


Now we want to find a polynomial differential system of degree 9 which has as invariant straight lines the straight lines of Figure 3. Working as in the case of Figure 1, such a polynomial differential system is:

$$
\begin{aligned}
x^{\prime}= & x\left(x^{2}-1\right)\left(x^{2}-(\sqrt{2}+1)^{2}\right)[4(3+2 \sqrt{2}) \\
& \left.-4(2+\sqrt{2}) x^{2}-12(2+\sqrt{2}) y^{2}+x^{4}-6 x^{2} y^{2}+21 y^{4}\right], \\
y^{\prime}= & y\left(y^{2}-1\right)\left(y^{2}-(\sqrt{2}+1)^{2}\right)[4(3+2 \sqrt{2}) \\
& \left.-12(2+\sqrt{2}) x^{2}-4(2+\sqrt{2}) y^{2}+21 x^{4}-6 x^{2} y^{2}+y^{4}\right] .
\end{aligned}
$$

If we want to find a polynomial differential system of degree 11 which has as invariant straight lines the straight lines of Figure 4. Working as in the case of Figure 1 we find that the polynomial differential system of degree 11 is:

$$
\begin{aligned}
x^{\prime}= & 8 x\left[-8(123+55 \sqrt{5})+220(47+21 \sqrt{5})\left(x^{2}+y^{2}\right)\right. \\
& +660(29+13 \sqrt{5})\left(x^{2} y-y^{3}\right)-1650(9+4 \sqrt{5})\left(x^{4}+2 x^{2} y^{2}+y^{4}\right) \\
& -(11+5 \sqrt{5})\left(-2145 x^{4} y+2970 x^{2} y^{3}+363 y^{5}\right) \\
& +825(7+3 \sqrt{5})\left(x^{6}+3 x^{4} y^{2}+3 x^{2} y^{4}+y^{6}\right) \\
& +330(2+\sqrt{5})\left(5 x^{6} y-5 x^{4} y^{3}-9 x^{2} y^{5}+y^{7}\right) \\
& -55(3+\sqrt{5})\left(5 x^{8}+90 x^{4} y^{4}-24 x^{2} y^{6}+9 y^{8}\right) \\
& \left.+2\left(25 x^{10}-275 x^{8} y^{2}+1650 x^{6} y^{4}-990 x^{4} y^{6}+165 x^{2} y^{8}+33 y^{10}\right)\right] \\
y^{\prime}= & (1-\sqrt{5}+4 y)(3+\sqrt{5}+2 y)(-2-\sqrt{5}+2 y)(1+\sqrt{5}+4 y) \\
& {\left[8(11+5 \sqrt{5}) y+40(7+3 \sqrt{5}) y^{2}-40(2+\sqrt{5})\left(11 x^{2} y-y^{3}\right)\right.} \\
& -5(3+\sqrt{5})\left(33 x^{4}+22 x^{2} y^{2}+5 y^{4}\right) \\
& \left.+2\left(165 x^{6}-165 x^{4} y^{2}+55 x^{2} y^{4}+y^{6}\right)\right] .
\end{aligned}
$$

Now we want to find a polynomial differential system of degree 13 which has as invariant straight lines the straight lines of Figure 7 . Working as in the case of Figure 1, such a polynomial differential system is:

$$
\begin{aligned}
x^{\prime}= & 2 x\left(x^{2}-3\right)\left(4 x^{2}-3\right)\left(4 x^{2}-27\right)(-324 \\
& +1011 x^{2}+1599 y^{2}-118 x^{4}+4888 x^{2} y^{2}-4290 y^{4} \\
& \left.+3 x^{6}-195 x^{4} y^{2}-2431 x^{2} y^{4}+1287 y^{6}\right), \\
y^{\prime}= & -y\left(4 y^{2}-1\right)\left(157464-777114 x^{2}-147258 y^{2}\right. \\
& -482625 x^{4}+1061424 x^{2} y^{2}-44631 y^{4} \\
& +389961 x^{6}-34749 x^{4} y^{2}-329589 x^{2} y^{4}+41553 y^{6}
\end{aligned}
$$




$$
\begin{aligned}
& -99099 x^{8}+28314 x^{6} y^{2}-30888 x^{4} y^{4}+48438 x^{2} y^{6}-7533 y^{8} \\
& +5005 x^{10}+9009 x^{8} y^{2}-12870 x^{6} y^{4}+7722 x^{4} y^{6} \\
& \left.-3159 x^{2} y^{8}+405 y^{10}\right)
\end{aligned}
$$

We want to remark that this polynomial differential system of degree 13 has "only" 30 invariant straight lines while from Lemma 9 we can produce a polynomial differential system of degree 13 with 32 invariant straight lines from a polynomial differential system of degree 11 . The difference is that the former has a finite number of singular points while the second has infinitely many singular points.

If we want to find a polynomial differential system of degree 15 which has as invariant straight lines the straight lines of Figure 5. Working as in the case of Figure 1, we find that such a polynomial differential system is:

$$
\begin{aligned}
x^{\prime}= & x(-\sqrt{2}+\sqrt{6}-4 x)(-\sqrt{2}+\sqrt{6}+4 x) \\
& {\left[19-11 \sqrt{3}-2\left((98-57 \sqrt{3}) x^{2}-9(12-7 \sqrt{3}) y^{2}\right)\right.} \\
& +2\left((355-209 \sqrt{3}) x^{4}+2(401-237 \sqrt{3}) x^{2} y^{2}+91(5-3 \sqrt{3}) y^{4}\right) \\
& -4\left((263-160 \sqrt{3}) x^{6}-(923-566 \sqrt{3}) x^{4} y^{2}\right. \\
& \left.-13(85-52 \sqrt{3}) x^{2} y^{4}-143(3-2 \sqrt{3}) y^{6}\right) \\
& +(551-375 \sqrt{3}) x^{8}+4(695-451 \sqrt{3}) x^{6} y^{2} \\
& +130(37-29 \sqrt{3}) x^{4} y^{4}+286(18-10 \sqrt{3}) x^{2} y^{6} \\
& +1287(1-\sqrt{3}) y^{8}-2\left((20-31 \sqrt{3}) x^{10}\right. \\
& -3(378-163 \sqrt{3}) x^{8} y^{2}+10(520-167 \sqrt{3}) x^{6} y^{4} \\
& \left.-286(30-11 \sqrt{3}) x^{4} y^{6}+429(4-\sqrt{3}) x^{2} y^{8}-143(2-3 \sqrt{3}) y^{10}\right) \\
& -4\left(3 x^{12}+192 x^{10} y^{2}-1235 x^{8} y^{4}+1144 x^{6} y^{6}\right. \\
& \left.\left.+1287 x^{4} y^{8}-1144 x^{2} y^{10}+143 y^{12}\right)\right], \\
y^{\prime}= & 4 y(-\sqrt{2}+\sqrt{6}-4 y)(-\sqrt{2}+\sqrt{6}+4 y) \\
& -19+11 \sqrt{3}+2\left(9(12-7 \sqrt{3}) x^{2}+(98-57 \sqrt{3}) y^{2}\right) \\
& -2\left(91(5-3 \sqrt{3}) x^{4}-2(401-237 \sqrt{3}) x^{2} y^{2}-(355-209 \sqrt{3}) y^{4}\right) \\
& +4\left(143(3-2 \sqrt{3}) x^{6}+(923-566 \sqrt{3}) x^{2} y^{4}\right. \\
& \left.+13(85-52 \sqrt{3}) x^{4} y^{2}+(263-160 \sqrt{3}) y^{6}\right) \\
& -1287(1-\sqrt{3}) x^{8}-572(9-5 \sqrt{3}) x^{6} y^{2}-130(37-29 \sqrt{3}) x^{4} y^{4} \\
& -4(695-451 \sqrt{3}) x^{2} y^{6}-(551-375 \sqrt{3}) y^{8} \\
& +2\left(143(2-3 \sqrt{3}) x^{10}-429(4-\sqrt{3}) x^{8} y^{2}+286(30-11 \sqrt{3}) x^{6} y^{4}\right.
\end{aligned}
$$




$$
\begin{aligned}
& \left.-130(40-9 \sqrt{3}) x^{4} y^{6}+3(378-163 \sqrt{3}) x^{2} y^{8}+(20-31 \sqrt{3}) y^{10}\right) \\
& -4\left(143 x^{12}+1144 x^{10} y^{2}-1287 x^{8} y^{4}-1144 x^{6} y^{6}\right. \\
& \left.\left.+1235 x^{4} y^{8}-192 x^{2} y^{10}+3 y^{12}\right)\right] .
\end{aligned}
$$

Finally, we wish to find a polynomial differential system of degree 19 which has as invariant straight lines the straight lines of Figure 8. Working as in the case of Figure 1, such a polynomial differential system is:

$$
\begin{aligned}
& x^{\prime}=2 x\left(4 x^{2}-3\right)\left(x^{2}-3\right)\left(4 x^{2}-27\right)\left(x^{2}-12\right) \\
& {\left[-476928+1831632 x^{2}+2736912 y^{2}\right.} \\
& -1236176 x^{4}+3156432 x^{2} y^{2}-7845024 y^{4} \\
& +101859 x^{6}-38019 x^{4} y^{2}-3200607 x^{2} y^{4}+5968071 y^{6} \\
& -2898 x^{8}+47196 x^{6} y^{2}-2062032 x^{4} y^{4} \\
& +3135684 x^{2} y^{6}-1687998 y^{8} \\
& +27 x^{10}-1197 x^{8} y^{2}+44574 x^{6} y^{4} \\
& \left.+398582 x^{4} y^{6}-466089 x^{2} y^{8}+138567 y^{10}\right] \text {, } \\
& y^{\prime}=y\left(4 y^{2}-1\right)\left(y^{2}-1\right) \\
& {\left[-2781444096+15961670784 x^{2}+2054450304 y^{2}\right.} \\
& -5944652208 x^{4}-11696006016 x^{2} y^{2}-469635408 y^{4} \\
& -531353088 x^{6}+4921574256 x^{4} y^{2}+2210841216 x^{2} y^{4} \\
& +85920912 y^{6}-1044278703 x^{8}+1567739286 x^{6} y^{2} \\
& -1800119052 x^{4} y^{4}+47250378 x^{2} y^{6}-27450981 y^{8} \\
& +324385347 x^{10}-225498897 x^{8} y^{2}-132437106 x^{6} y^{4} \\
& +233426286 x^{4} y^{6}-43256673 x^{2} y^{8}+4751379 y^{10} \\
& -35519341 x^{12}+24387792 x^{10} y^{2}-8276229 x^{8} y^{4} \\
& +18209448 x^{6} y^{6}-16665831 x^{4} y^{8}+3878280 x^{2} y^{10}-341415 y^{12} \\
& +969969 x^{14}+877591 x^{12} y^{2}-2909907 x^{10} y^{4}+2607579 x^{8} y^{6} \\
& \left.-1508733 x^{6} y^{8}+601749 x^{4} y^{10}-115425 x^{2} y^{12}+8505 y^{14}\right] \text {. }
\end{aligned}
$$

Proof of Theorem 8. By using Lemma 9 and the polynomial differential systems listed above, which have as invariant straight lines those of Figures 1 to 8 , Theorem 8 follows at once.

Unfortunately, we cannot compute the polynomial differential systems of degree $n \in\{25,31, \ldots\}$ having as invariant straight lines the lines of the arrangements $A(2 n+5)$. Even if we could compute them one by one, we doubt that we could find a relationship among their coefficients and their 
degree. If we were able to do so, we would have counterexamples to the Invariant Straight Line Conjecture for arbitrarily large degrees of the form $n=25,31, \ldots$

Remarks. 1. Obviously for every polynomial differential system all the intersections among invariant straight lines are finite singular points. But we want to point out that this does not imply at all that all finite singular points must be the intersection of invariant straight lines; in fact, examples to this effect are known.

2. The determination of the exact values of $\alpha(n)$ and $\gamma(n)$ seems to be a very challenging and hard task. This is made strikingly evident by the absence of any specific conjecture concerning these values. On the other hand, all available information is consistent with the following open questions:

Question 1. $\alpha(n)=\gamma(n)$ for all $n \geq 1$ ?

Question 2. $\lim _{n \rightarrow \infty} \frac{\alpha(n)}{n}=\lim _{n \rightarrow \infty} \frac{\gamma(n)}{n}=2$ as $n \rightarrow \infty$ ?

\section{References}

[ALGM] A.A. Andronov, E.A. Leontovich, I.I. Gordon and A.G. Maier, Qualitative theory of second order dynamic systems, John Wiley \& Sons, 1973.

[Ca] M.P. do Carmo, Differential geometry of curves and surfaces, Prentice-Hall, Inc., 1976.

[CF] G.E. Cooke and R.L. Finney, Homology of cell complexes, Mathematical Notes, Princeton Univ. Press, 1967.

[Da] G. Darboux, Mémoire sur les équations différentielles algébriques du premier ordre et du premier degré, Bull. Sciences Math. Sér. (2), 2 (1878), 60-96, 123-144, 151-200.

[Go] E.A. González, Generic properties of polynomial vector fields at infinity, Trans. Amer. Math. Soc., 143 (1969), 201-222.

[Gr] B. Grünbaum, Arrangements of hyperplanes, Proc. of the Second Louisiana Conference on Combinatorics, Graph Theory and Computing, Louisiana State University, Baton Rouge, (1971), 41-106.

[Ma] W.S. Massey, Singular homology theory, Graduate Texts in Math., 70, SpringerVerlag, 1980.

[P1] H. Poincaré, Mémoire sur les courbes définies par les équations différentielles, J. Math. Pures Appl. Sér. 3, 7 (1881), 375-422.

[P2] - Mémoire sur les courbes définies par les équations différentielles, J. Math. Pures Appl. Sér. 3, 8 (1882), 251-296.

[P3] Mémoire sur les courbes définies par les équations différentielles, J. Math. Pures Appl. Sér. 4, 1 (1885), 167-244.

[Sc] D. Schlomiuk, Algebraic and geometric aspects of the theory of polynomial vector fields, in 'Bifurcations and periodic orbits of vector fields', edited by D. Schlomiuk, Kluwer Acad. Publ., (1993), 429-467. 
[Sk] J. Sokulski, On the number of invariant lines of polynomial vector fields, Nonlinearity, 9 (1996), 479-485.

[So] J. Sotomayor, Curves definides per equaçóes diferenciais no plano, Instituto de Matemática Pura e Aplicada, Rio de Janeiro, 1979.

[Sp] E.H. Spanier, Algebraic topology, McGraw-Hill, 1966.

[SS] Suo Guangjian and Sun Jifang, The $n$-degree differential system with $(n-1)(n+$ 2)/2 straight line solutions has no limit cycles, in 'Proc. of Ordinary Differential Equations and Control Theory', Wuhan (in Chinese), (1987), 216-220.

[Zh] Zhang Xikang, Number of integral lines of polynomial systems of degree three and four, J. of Nanjing University, Math. Biquartely, 10 (1993), 209-212.

[Zo] H. Zoladek, Private communication, 1995.

Received June 3, 1996 and revised June 26, 1997.

Universitat Autònoma de Barcelona

Bellaterra 08193, Barcelona, Spain

E-mail address: artes@mat.uab.es

UNIVERSITY OF WASHINGTON

SEATTLE WA 98195-4350

AND

Universitat Autònoma de Barcelona

Bellaterra 08193, BArCelona, Spain

E-mail address: jllibre@mat.uab.es 